\title{
Aplicação da espectroscopia Raman na identificação de olivinas em meteoritos
}

\author{
Bruno Leonardo do Nascimento-Dias ${ }^{1 *}$ e Virgilio de Carvalho dos Anjos ${ }^{1}$
}

${ }^{1}$ Grupo de Engenharia e Espectroscopia de Materiais, Universidade Federal de Juiz de Fora, Departamento de Física, Juiz de Fora, Minas Gerais, Brasil.

\section{DOI: https://doi.org/10.30609/jeti.v4i2.12434}

\begin{abstract}
Raman spectroscopy technique has been increasingly used to characterize terrestrial rocks and meteorites in general. However, the literature in Portuguese with information on how to distinguish the minerals that make up rocks, whether terrestrial or extraterrestrial, is scarce and almost nonexistent. Thus, the objective of this work is to present how it is possible to determine the presence of olivines and to identify which type of mineral in the group is specifically found in the sample in a quick, succinct and accessible way. It is worth noting that the olivine group is characterized by its final members, fayalite $\left(\mathrm{Fe}_{2} \mathrm{SiO}_{4}\right)$ and forsterite $\left(\mathrm{Mg}_{2} \mathrm{SiO}_{4}\right)$. The identification of the spectra was carried out from spectra of pure minerals allocated in the RRUFF database and compared with spectra obtained from the carbonaceous meteorites (Allende and Murchison) and the Martian meteorite NWA 7397. Finally, this work does not have the purpose of having a detailed deepening of analyzes and the greater specificities, which can be acquired by readers through complementary materials and with the assistance of more specialized technicians in Raman's equipment and technique.
\end{abstract}

Keywords: Fayalite, Forsterite, Meteorite, Olivine, Raman 
Resumo. A técnica de espectroscopia Raman tem sido cada vez mais utilizada na caracterização de rochas terrestres e meteoritos em geral. No entanto, a literatura em língua portuguesa com informações sobre como se distinguir os minerais constituintes de rochas, sejam terrestres ou extraterrestres, são escassos e quase inexistentes. Dessa forma, o objetivo deste trabalho é apresentar como é possível determinar a presença de olivinas e identificar qual tipo de mineral do grupo especificamente se encontra na mostra de maneira rápida, sucinta e acessível. Vale salientar que o grupo das olivinas é caracterizado pelos seus membros finais, a faialita $\left(\mathrm{Fe}_{2} \mathrm{SiO}_{4}\right)$ e a forsterita $\left(\mathrm{Mg}_{2} \mathrm{SiO}_{4}\right)$. A identificação dos espectros foi realizada a partir de espectros de minerais puros alocados no banco de dados RRUFF e comparados com espectros obtidos dos meteoritos carbonáceos (Allende e Murchison) e o meteorito marciano NWA 7397. Por fim, este trabalho não possui o propósito de ter um aprofundamento detalhado de análises e as especificidades maiores do Raman, o qual poderá ser adquirido pelos leitores através de materiais complementares e com assistências de técnicos mais especializados no equipamento e na técnica de Raman.

Palavras-chaves: Faialita, Forsterita, Meteorito, Olivina, Raman.

\section{Introduction}

As olivinas são um grupo de minerais formadores de rocha caracterizados pela fórmula química $(\mathrm{Fe}, \mathrm{Mg})_{2} \mathrm{SiO}_{4}$. Elas variam em sua composição pela quantidade de ferro $(\mathrm{Fe})$ e magnésio $(\mathrm{Mg})$ presentes na sua estrutura química. A série mais comum neste sistema é a da forsterita $\left(\mathrm{Mg}_{2} \mathrm{SiO}_{4}\right)$ até a faialita $\left(\mathrm{Fe}_{2} \mathrm{SiO}_{4}\right)$ [1]. Esse grupo de minerais formadores de rochas são considerados minerais essenciais, ou seja, minerais como as olivinas compõem a maioria da mineralogia da rocha, sendo assim, definem, classificam e caracterizam grande parte das rochas terrestres e rochas extraterrestres [2]. Em geral, são encontradas principalmente em rochas ígneas ricas em magnésio $(\mathrm{Mg})$, tais como gabro, peridotito e basalto, e coexistindo com plagioclásios e piroxênios [3].

A técnica de espectroscopia Raman tem sido cada vez mais utilizada na caracterização de rochas terrestres e meteoritos em geral [4-6]. As investigações estão se tornando ainda mais precisas com o passar dos tempos devido aos recursos de microfocalização, de modo a ser possível identificar diferentes formas cristalinas que podem compor as amostras [7-8]. Dessa forma, é cada vez mais imprescindível informações sobre como se distinguir os minerais constituintes de rochas, sejam terrestres ou extraterrestres, através desta técnica analítica. Vale salientar que a espectroscopia Raman é uma técnica analítica que possui grande eficiência, com rápida aquisição de dados, além de ser um 
método não invasivo. Sendo assim, poderia ser utilizada como análise preliminar em amostras raras ou que se tenha pouco material disponível para ser examinado por outras técnicas [9].

Os modos vibracionais obtidos através da espectroscopia Raman são particulares e característicos. Neste sentido, um espectro de Raman típico das olivinas possui 2 picos característicos e aqui serão chamados de picos A e B. O primeiro pico (A), se encontra na faixa entre $814-824 \mathrm{~cm}^{-1}$, enquanto o pico $\mathrm{B}$, fica na faixa de $836-857 \mathrm{~cm}^{-1}$ [10-12]. É importante frisar que as olivinas possuem 84 modos vibracionais, entretanto apenas 36 são considerados modos vibracionais ativos para o Raman [13-14].

Em um espectro de Raman varrendo $200-4000 \mathrm{~cm}^{-1}$ é possível encontrar outros picos além dos já mencionados picos A e B. Na região do espectro entre 400-700 $\mathrm{cm}^{-1}$ é normal encontrar alguns picos, sendo estes resultados de modos vibracionais de flexões internas do grupo iônico SiO4. Por vezes são detectados picos abaixo dos $400 \mathrm{~cm}^{-1}$ e estes picos são atribuídos aos modos de rede: movimentos de rotação e translação de $\mathrm{SiO} 4$ vinculados aos movimentos de translação de cátions octaédricos $\left(\mathrm{Mg}^{+2}, \mathrm{Fe}^{+2}\right)$ na estrutura de cristalográfica [12].

Por fim, a proposta deste texto é apresentar uma breve síntese sobre como determinar e identificar as olivinas detectadas em espectros de Raman. O principal motivo se dá por conta de não existir literatura em português disponível voltada para este tipo de pesquisa e analogias com fácil acesso. Em geral, as especificidades de técnicas analíticas muitas vezes impedem que o leitor iniciante (da graduação e, por vezes, da pós-graduação) possa perceber sua aplicabilidade e até mesmo como interpretar seus resultados. Assim, este trabalho não possui o propósito ter um aprofundamento detalhado de análises e as especificidades maiores poderão ser adquiridas pelos leitores através de materiais complementares e com assistências de técnicos mais especializados no equipamento e na técnica de Raman.

\section{Materiais e Métodos}

Os materiais a serem analisados neste artigo serão meteoritos já estudados anteriormente e com artigos publicados pelo autor. Serão escolhidos espectros de olivinas que foram detectadas nesses meteoritos. Os espectros foram obtidos através do equipamento Confocal SENTERRA BRUKER da Universidade Federal de Juiz de Fora (UFJF). O instrumento é equipado com uma câmera de CCD (ANDOR DU420-OE) e possui uma resolução espectral de aproximadamente $4 \mathrm{~cm}^{-1}$ e possui uma área de atuação entre $100-4000 \mathrm{~cm}^{-1}$. Possui 
calibração automática contínua $\left(0.1 \mathrm{~cm}^{1}\right.$ acurácia teórica). As análises foram adquiridas usando uma lente objetiva do microscópio Raman de 50x e foi utilizado um laser de 632.8 $\mathrm{nm}$ laser e padronizada uma energia de excitação de $5 \mathrm{~mW}$. Antes de inicar todas as aquisições foi realizada a calibração por meio de uma pastilha padrão de silício que possui um pico característico de $521 \mathrm{~cm}^{-1}$. Todos os espectros de Raman foram processados através do software OriginPro8@.

O método escolhido para identificar as olivinas será o de comparação analítica entre espectros obtidos com os de minerais puros. Assim, através do banco de dados de espectros de Raman de minerais puros encontradas no RRUFF serão feitas as identificações dos minerais presentes nos meteoritos. Esta abordagem escolhida para este trabalho é eficiente, rápida e além de prática, também é de fácil compreensão para leitores iniciantes na técnica.

\section{Resultados e Discussões}

Apesar da simplicidade com que a espectroscopia Raman será abordada aqui, estudos mais técnicos e específicos mostram que essa técnica, quando usada em sua plenitude, fornece subsídios para caracterização detalhada de inúmeros materiais. Para a demonstração dos resultados, os dados de olivinas foram divididos em 2: espectro de Forsterita e outro contendo espectro de Faialita. As análises dos espectros foram apresentadas de forma a evidenciar o comportamento geral de cada um dos minerais de Olivina.

\subsection{Faialita}

A faialita é um mineral do grupo das olivinas e a sua composição química é $\mathrm{Fe}_{2} \mathrm{SiO}_{4}$. Os picos que caracterizam a faialita são respectivamente, pico A entre $814-820 \mathrm{~cm}^{-1}$ e o pico B entre 845-851 $\mathrm{cm}^{-1}$. Na Figura 1 é possível observar os picos A e B obtidos através da análise do meteorito Allende, que é um condrito carbonáceo do tipo CV3. O primeiro pico se encontra em $818 \mathrm{~cm}^{-1}$ e o segundo em $848 \mathrm{~cm}^{-1}$. Na Figura 2 aparece outro espectro em que os picos A e B, respectivamente, aparecem em $820 \mathrm{~cm}^{-1}$ e $850 \mathrm{~cm}^{-1}$, os quais foram obtidos a partir da análise do meteorito Murchison, que é um meteorito carbonáceo do tipo CM2.

O espectro de mineral puro de faialita (ID: X050076) do site RRUFF foi posto na Figura 3 para ser comparado com os espectros de Raman obtidos dos meteoritos Murchison e Allende. Dessa forma, através da metodologia comparativa analítica é possível atribuir a presença da olivina em ambos os meteoritos, tal como confirmar a identificação e presença de faialita. 


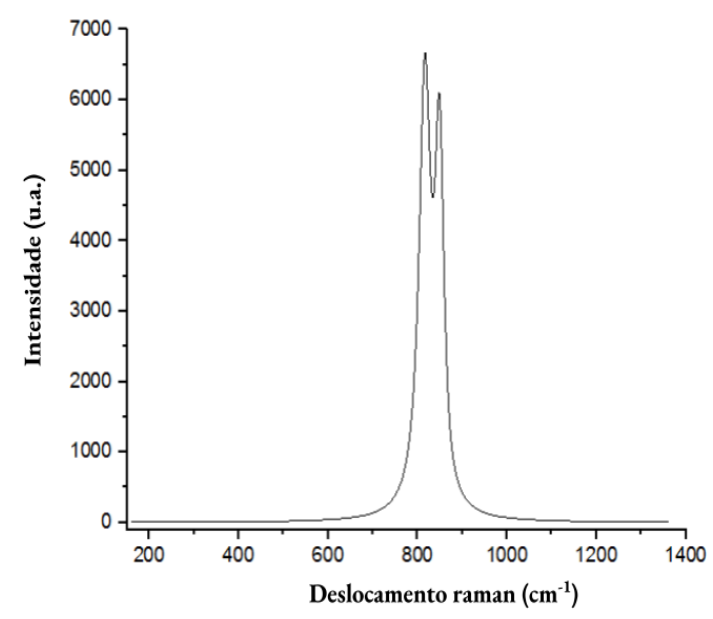

Figura 1 - Espectro de Raman do meteorito Allende

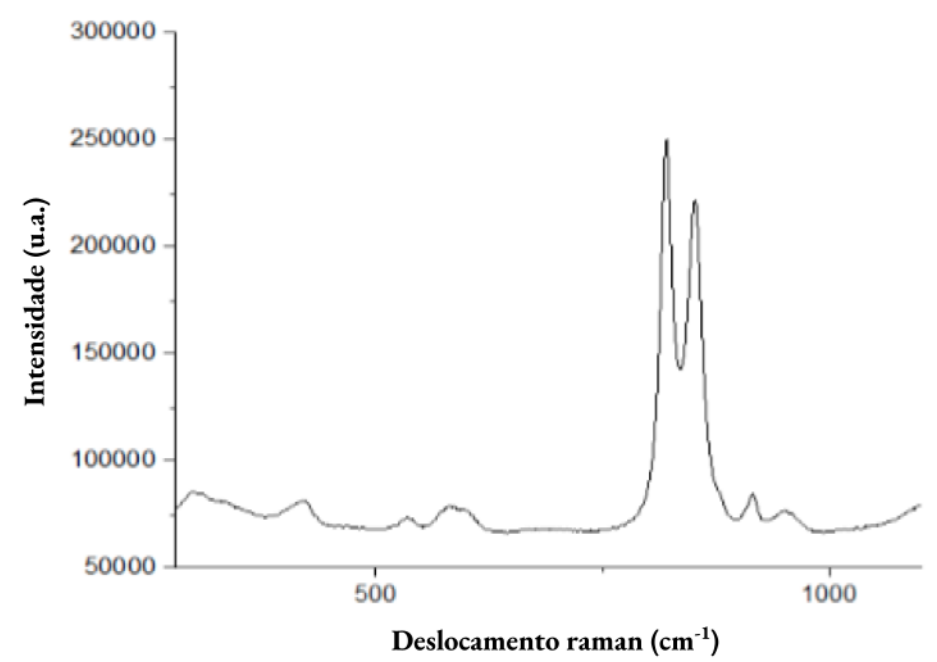

Figura 2 - Espectro de Raman do meteorito Murchison

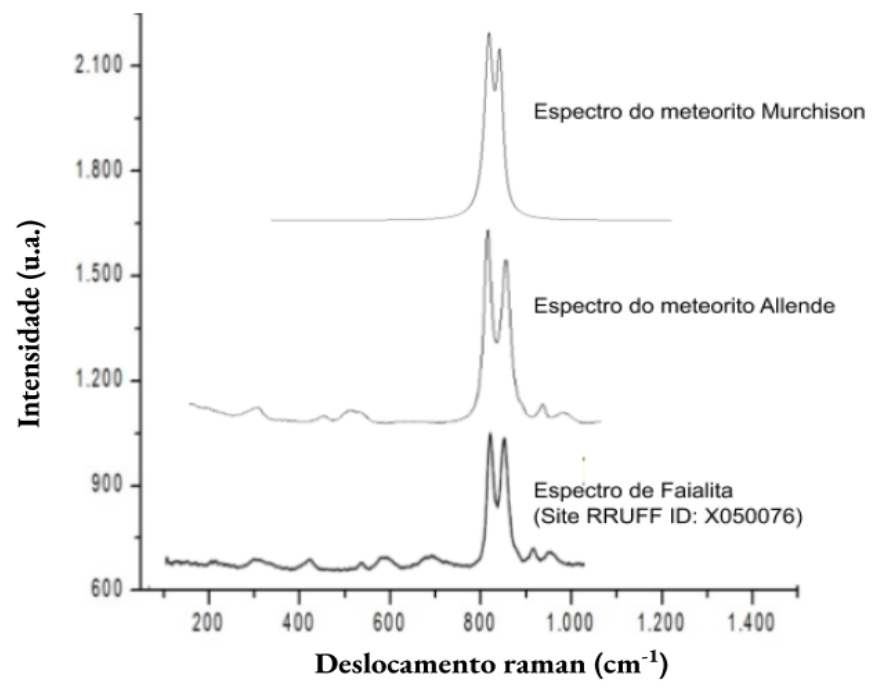

Figura 3 - Comparativo entre os espectros de Raman obtidos e o de mineral puro de Faialita 


\subsection{Forsterita}

A forsterita é outro um mineral integrante do grupo das olivinas e a sua composição química é $\mathrm{Mg}_{2} \mathrm{SiO}_{4}$. Os picos são muito semelhantes aos da faialita, porém a forsterita possui respectivamente, pico A entre $825-830 \mathrm{~cm}^{-1}$ e o pico $\mathrm{B}$ entre $852-858 \mathrm{~cm}^{-1}$. O meteorito marciano NWA 7397 foi analisado pela técnica de Raman e na Figura 4 é apresentado o espectro obtido, em que os picos A e B aparecem. O primeiro pico se encontra em $826 \mathrm{~cm}^{-1}$ e o segundo em $853 \mathrm{~cm}^{-1}$. O espectro de mineral puro de forsterita (ID: X050088) do site RRUFF foi posto na Figura 5 para ser comparado com o espectro do meteorito marciano. Assim, é possível atribuir não apenas a presença da olivina no meteorito NWA 7397, tal como identificá-la como forsterita.

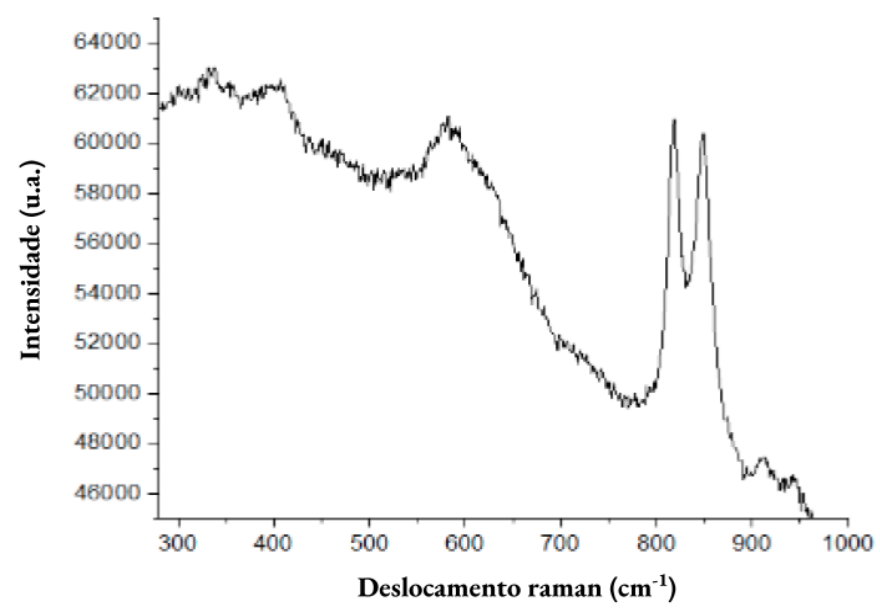

Figura 4 - Espectro de Raman do meteorito Murchison

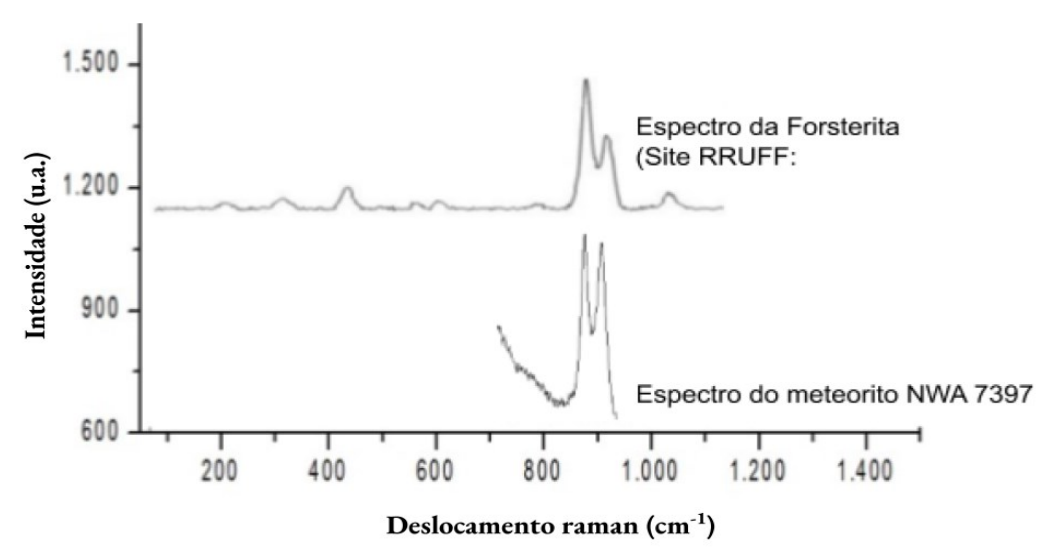

Figura 5 - Comparativo entre os espectros do NWA 7397 e o de mineral puro de forsterita.

Esta é uma forma rápida de se realizar atribuições mineralógicas, por exemplo, a uma rocha analisada por raman. De modo resumido, é possível observar que os picos 
correspondentes às bandas do mineral piroxênio da literatura coincidem com as bandas obtidas no espectro do meteorito Zagami. Nem todo espectro possui essa característica de fácil identificação, podem haver casos em que exista sobreposição de picos, dois ou mais minerais presentes na amostra. Nesses casos, a experiência da pessoa que está manuseando os dados e a sensibilidade do instrumento utilizado são de extrema relevância para obtenção de uma conclusão bem-sucedida.

\section{Conclusão}

Em geral, áreas como a Geologia, Ciências Planetárias, Meteorítica e Astrobiologia, por vezes, precisam e utilizam informações de rochas extraterrestres. Os meteoritos, embora sejam rochas extraterrestres, como qualquer outra rocha terrestre, são feitos de um conjunto de minerais. Assim, devido a raridade pode ser concluído que a técnica de Raman possui boa eficiência na detecção de minerais. Além disso, as interpretações preliminares para alguns grupos podem ser obtidas através de comparações com espectros da literatura de minerais puros. Particularmente, neste trabalho foi possível verificar e concluir a viabilidade de determinar a presença de olivina em meteoritos, tal como identificar o membro do grupo. A caracterização qualitativa é simples e rápida, de modo não invasivo pode ser diferenciado os minerais Forsterita e Faialita, ambos constituintes do grupo das Olivinas.

Espera-se que este artigo possa ser utilizado como referência para alunos de graduação e Pós-graduação, principalmente, por aqueles que estejam mexendo pela primeira vez com esta técnica analítica para essas finalidades. Assim, este é um trabalho que busca servir como referência inicial para o desenvolvimento e aprendizagem do Raman, seus conceitos para futuros trabalhos de iniciação científica, monografias, dissertações ou até mesmo teses.

\section{Agradecimentos}

Os autores agradecem as agências CAPES e ao Cnpq pelo fomento oferecido para desenvolver essa pesquisa, tal como aos revisores pelas contribuições para o aprimoramento do conteúdo.

\section{Referências Bibliográficas}

[1] KLEIN, C.; DUTROW, B. Manual de Ciências dos Minerais (23ํ edição). Tradução e revisão técnica: Rualdo Menegat. Editora Bookman, Porto Alegre, Brasil, 2012. 
[2] ZUCOLOTTO, Maria Elizabeth et al. Decifrando os meteoritos. Universidade Federal do Rio de Janeiro, Museu Nacional, 2013.

[3] DEER, W. A.; HOWIE, R. A.; ZUSSMAN, J. Minerais Constituintes das Rochas-Uma Introdução-2a edição. Fundação Calouste Gulbenkian. Lisboa, 2008.

[4] DO NASCIMENTO-DIAS, Bruno Leonardo et al. Probing the chemical and mineralogical characteristics of the Martian meteorite NWA 7397 through $\mu$ Raman and $\mu$ XRF non-destructively. International Journal of Astrobiology, v. 18, n. 1, p. 73-78, 2019.

[5] DO NASCIMENTO-DIAS, Bruno Leonardo et al. Detection of organic or inorganic material in Martian meteorite Zagami by vibrational spectroscopy?. International Journal of Astrobiology, v. 19, n. 6, p. 438-445, 2020.

[6] SERBESTOGLU, M.; YESILTAS, M.; YALCIN, M. Gurhan. Mineralogy of Northwest Africa 6963 Determined by Raman Spectroscopy. In: METEORITICS \& PLANETARY SCIENCE. 111 RIVER ST, HOBOKEN 07030-5774, NJ USA: WILEY, 2019.

[7] RODRIGUES, A. DE G.; GALZERANI, J. C. Espectroscopias de infravermelho, Raman e de fotoluminescência: potencialidades e complementaridades. Revista Brasileira de Ensino de Física, v. 34, n. 4, p. 1-9, 2012.

[8] LOBO, A. O. et al. Caracterização de materiais carbonosos por espectroscopia Raman. Revista Brasileira de Aplicações de Vácuo, v. 24, n. 2, p. 98-103, 2008.

[9] NASCIMENTO-DIAS, Bruno. Raman spectroscopy and its peculiarities. Journal of Experimental Techniques and Instrumentation, v. 4, n. 01, p. 15-25, 2020.

[10] KUEBLER, Karla E. et al. Extracting olivine (Fo-Fa) compositions from Raman spectral peak positions. Geochimica et Cosmochimica Acta, v. 70, n. 24, p. 6201-6222, 2006.

[11] DURBEN, Dan J.; MCMILLAN, Paul F.; WOLF, George H. Raman study of the highpressure behavior of forsterite (Mg2SiO4) crystal and glass. American Mineralogist, v. 78, n. 11-12, p. 1143-1148, 1993.

[12] CHOPELAS, A. Single crystal Raman spectra of forsterite, fayalite, and monticellite. American Mineralogist, v. 76, n. 7-8, p. 1101-1109, 1991.

[13] PITTARELLO, Lidia et al. Screening and classification of ordinary chondrites by Raman spectroscopy. Meteoritics \& Planetary Science, v. 50, n. 10, p. 1718-1732, 2015.

[14] MOHANAN, Kakkala; SHARMA, Shiv K.; BISHOP, Finley C. A Raman spectral study of forsterite-monticellite solid solutions. American Mineralogist, v. 78, n. 1-2, p. 4248, 1993. 